\title{
Di-jets: the Path to the (un)polarized Partonic Photon Structure at an EIC
}

\author{
X. Chu* \\ Key Laboratory of Quark and Lepton Physics (MOE) and \\ Insititute of Particle Physics, Central China Normal University, \\ Wuhan 430079, China \\ Physics Derpartment, Brookhaven National Laboratory, \\ Upton, New York 11973, USA \\ E-mail: xchu@mails.ccnu.edu.cn
}

\section{E.C. Aschenauer}

Physics Derpartment, Brookhaven National Laboratory,

Upton, New York 11973, USA

E-mail: elke@bnl.gov

\begin{abstract}
We first state that our presentation in DIS2018 conference includes two aspects of research: the underlying events and the photon structure which has been published in the earlier DIS proceeding in 2016, so in this proceeding, we will focus on the study of underlying events. In high energy physics, the underlying event is everything except the two outgoing hard scattered jets, it includes the contributions from beam remnant as well as initial and final-state radiation. The behavior of underlying event at a future Electron Ion Collider (EIC) is studied in this proceeding. We apply both "region method" and "off-axis method" to measure the underlying event with pseudo data generated from PYTHIA. It indicates that these two methods are consistent, and the final results are comparable with those measured from STAR at similar center-mass energy.
\end{abstract}

XXVI International Workshop on Deep-Inelastic Scattering and Related Subjects (DIS2018)

16-20 April 2018

Kobe, Japan

\footnotetext{
* Speaker.
} 


\section{Introduction}

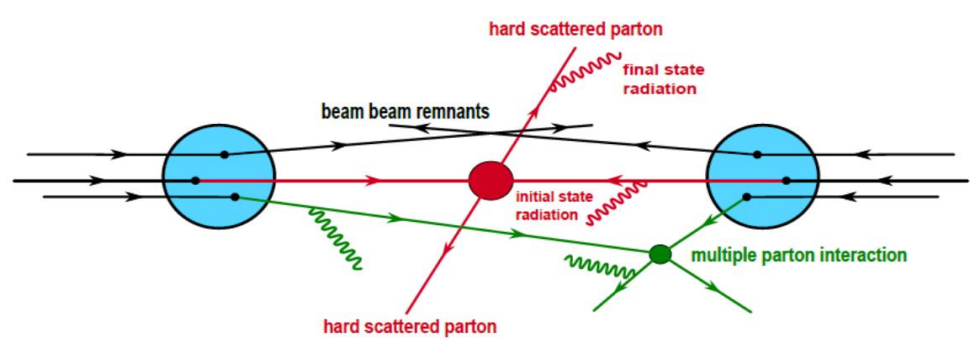

Figure 1: Illustration of underlying events.

Although the hardest 2-to-2 scattering is of the most interest, the other soft scatterings as background to the hard processes are also important to be studied. The background generated due to multiple soft scatterings is classified as underlying events. The underlying event is everything except the two outgoing hard scattered jets and consists of the beam remnant plus possible contributions from the "hard scattering" arising from the initial and final-state radiation. The underlying event contribution, as one of the background contributions to jet signals, is measured at a future Electron Ion Collider (EIC) in this proceeding. One possible design for an EIC is eRHIC [1] at Brookhaven National Laboratory (BNL). Di-jet method is applied in the analysis. High $p_{T}$ di-jet events belong to resolved, QCD Compton (QCDC) and Photon Gluon Fusion (PGF) processes from PYTHIA-6.4 [2] in $e p$ collision. In resolved processes, the QCD Monte Carlo models simulate a electron-proton collision through a hard parton+parton $\rightarrow$ parton+parton scattering. In QCDC and PGF, the collision occurred through a $\gamma+$ parton $\rightarrow$ parton+parton scattering. From both two types of scatterings, the resulting event contains particles from the two outgoing partons and those coming from the breakup of the incoming particles (beam remnants).

\section{Region method}

Two different methods are discussed to measure the underlying events in $e p$ collision. The first method is called the "region method". In each high $p_{T}$ di-jet event, the highest $p_{T}$ jet is called the trigger jet, the second highest $p_{T}$ jet is called the associate jet, these two jets are almost back-to-back in $\phi$ direction. We use the direction of the trigger jet in each event to divide the particles into three regions in $\eta-\phi$ space, where $\eta$ is the pseudorapidity measured along the beam axis and $\Delta \phi\left(\phi-\phi_{\text {trig jet }}\right)$ is the azimuthal angle relative to the trigger jet. The "toward" region contains the trigger jet, while the "away" region, on the average, contains the associate jet. The "transverse" region is perpendicular to the plane of the hard 2-to-2 scattering and is very sensitive to the "underlying event" component of the QCD Monte Carlo models. Fig. 2 illustrates the way we define the three regions. The average number of charged particles, $\left\langle N_{c h}\right\rangle$, we also call it "multiplicity", and the average scalar $p_{T}$ sum of charged particles, $\left\langle p_{T}\right.$ sum $\rangle$, in each region are measured versus $\Delta \phi$. A di-jet is reconstructed in each event with stable particles, the particles inside the jets are required to be $p_{T}>250 \mathrm{MeV}$ and $-4.5<\eta<4.5$. The di-jet events are selected 

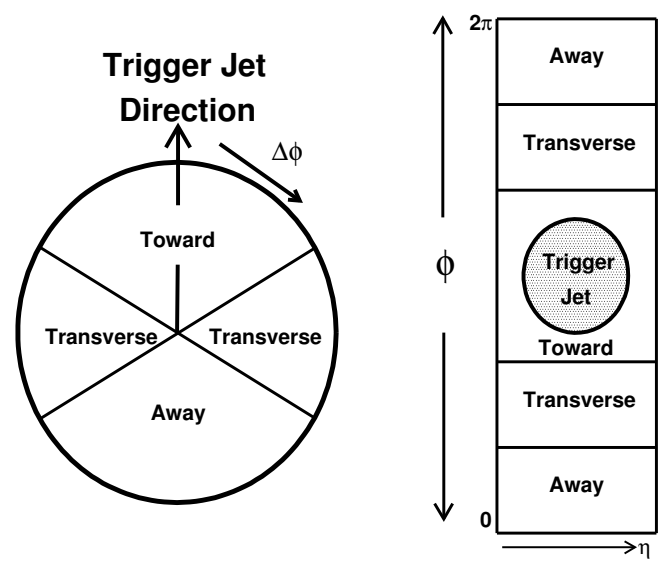

Figure 2: Illustration of "toward", "away" and "transverse" regions in azimuthal angle $\Delta \phi$ relative to the direction of the trigger jet in the event. The angle $\Delta \phi=\phi-\phi_{\text {trig jet }}$ is the relative azimuthal angle between charged particles and the trigger jet. The "toward" region is defined by $|\Delta \phi|<60^{\circ}$ (includes particles inside the trigger jet), while the "away" region is $|\Delta \phi|>120^{\circ}$. The "transverse" region is defined by $60^{\circ}<|\Delta \phi|<$ $120^{\circ}$. The plot is from [3].

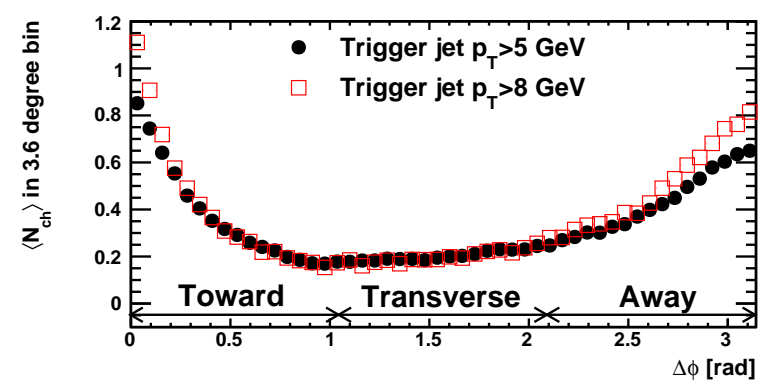

Figure 3: Average number of charged stable particles as a function of the azimuthal angle, $\Delta \phi$, between the particle and the trigger jet.

with the cut of $10^{-5} \mathrm{GeV}^{2}<Q^{2}<0.1 \mathrm{GeV}^{2}$. When we plot $\left\langle N_{c h}\right\rangle$ and $\left\langle p_{T}\right.$ sum $\rangle$ as a function of $\Delta \phi$, we include all the charged particles.

Fig. 3 and Fig. 4 show the results of the charged multiplicity distribution and the sum of transverse momentum distribution, respectively, in the azimuthal angle $\Delta \phi$ relative to the trigger jet for $p_{T}>5 \mathrm{GeV}$ and $8 \mathrm{GeV}$. The "toward" region contains the highest charged particle multiplicity and summation of charged particle transverse momentum. The associated jet is not absolutely back-to-back with the trigger jet in $\phi$ direction, which results in the long tails in the "away" region for both plots. In the "transverse" region where we put our interest of the underlying events, a relatively flat distribution is presented. Then the background effect due to the underlying events can be removed by subtraction of the multiplicity and summation of the transverse momentum in the "transverse" region. We calculate the average number of charged particles in middle pseudo rapidity in three different $Q^{2}$ bins to show the $Q^{2}$ dependence in Fig. 5. High $Q^{2}$ shows a much 


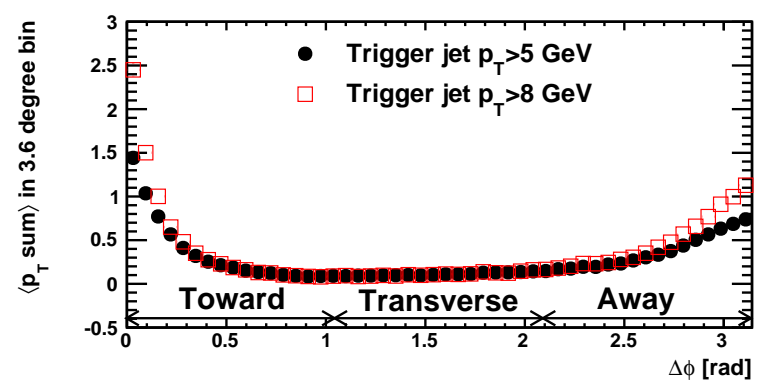

Figure 4: Average scalar $p_{T}$ sum of charged stable particles as a function of the azimuthal angle.

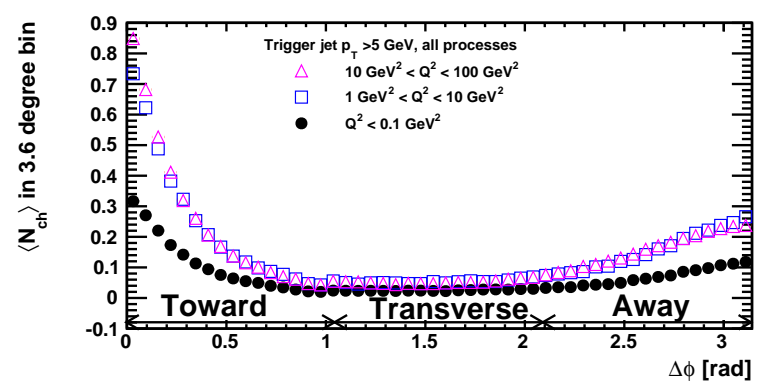

Figure 5: Average number of charged stable particles (middle pseodurapidity particles: $-1<\eta<1$ ) as a function of the azimuthal angle, $\Delta \phi$, between the particle and the trigger jet for $p_{T}^{\text {trig jet }}>5 \mathrm{GeV},-1<$ $\eta^{\text {trig jet }}<1$, in $Q^{2}<0.1 \mathrm{GeV}^{2}, 1<Q^{2}<10 \mathrm{GeV}^{2}$ and $10<Q^{2}<100 \mathrm{GeV}^{2}$ bins.

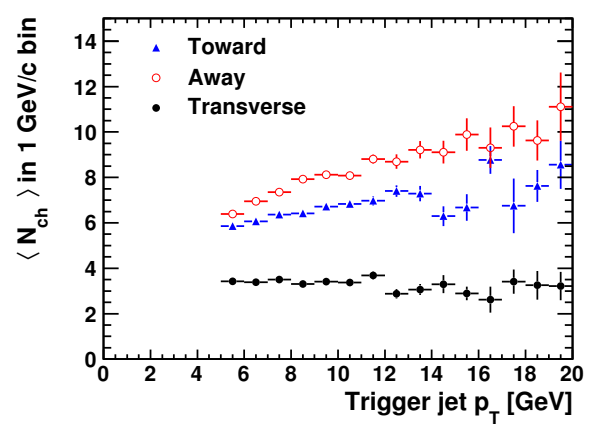

(a)

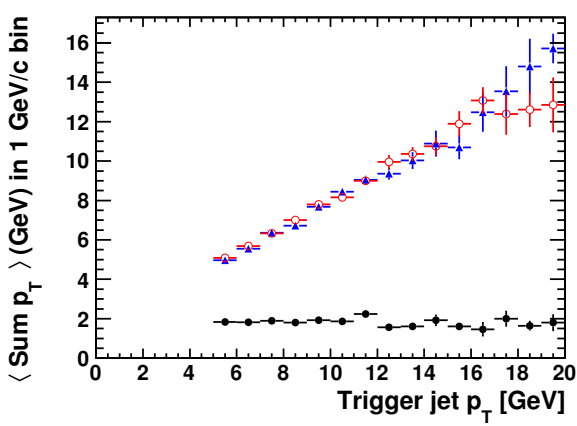

(b)

Figure 6: The average number and average scalar $p_{T}$ sum of charged particles in three regions.

higher number of charged particles than low $Q^{2}$, especially in "toward" and "away" regions, in the "transverse" region, a very small increase is observed when $Q^{2}$ is high. Fig. 6(a) shows the average number of charged particles in $1 \mathrm{GeV}$ bin as a function of the trigger jet $p_{T}\left(p_{T}>5 \mathrm{GeV}\right)$ in three regions. Fig. 6(b) shows the average scalar $p_{T}$ sum of charged particles $\left(p_{T}>250 \mathrm{MeV}\right.$ and $-4.5<\eta<4.5)$ in $1 \mathrm{GeV}$ bin as a function of the trigger jet $p_{T}\left(p_{T}>5 \mathrm{GeV}\right)$ in three regions. 


\section{Off-axis method}

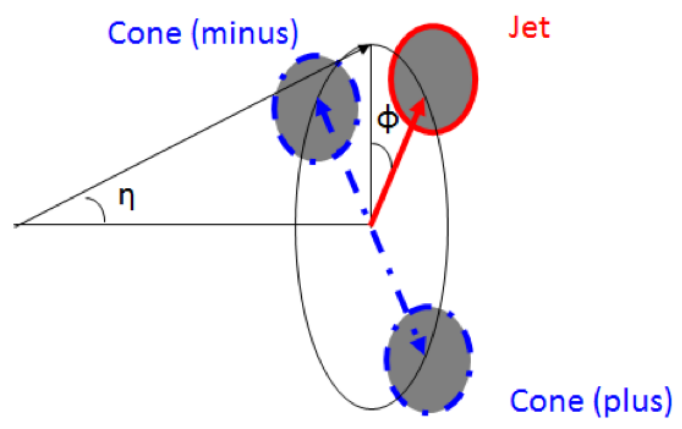

Figure 7: The illustration of two off-axis cones relative to a jet. The plot is from Ref. [4].

The second method is called "off-axis cone method" [5], which is developed by the ALICE experiment. The off-axis cone method is a method to study underlying event on the level of jet by jet, different from the region method which is on the level of event. For every reconstructed jet, we draw two off-axis cones (cone(-) and cone(+)), each of which is centered at the same $\eta$ as the jet but $\pm \pi / 2$ away in $\phi$ from the jet $\phi$ as shown in Fig. 7. Then we collect particles falling inside the two cones. The particle candidate pool consists of the input as used for the jet finding algorithm $\left(p_{T}>250 \mathrm{MeV}\right.$ and $-4.5<\eta<4.5$ ). We choose the cones radius $R$ to be 0.4 . The multiplicity density is defined as the average number of charged particles inside each cone $\left\langle N_{c h}\right\rangle$ divided by the cone area $\pi R^{2}$. The $\left\langle p_{T}\right.$ sum $\rangle$ density is defined as the average off-axis cone $p_{T}$ divided by the cone area.

We compare the results from the two methods in Fig. 8(a) and Fig. 8(b). Results from the region method are measured of all the charged particles in the "transverse" region with each particle in the pseudorapidity range from -1 to 1 and with the trigger jet pseudorapidity range as well as between -1 and 1 , so the multiplicity density $(\langle\mathrm{d} N\rangle / \mathrm{d} \phi \mathrm{d} \eta)$ and transverse momentum density $\left(\left\langle\mathrm{d} p_{T}\right.\right.$ sum $\left.\rangle / \mathrm{d} \phi \mathrm{d} \eta\right)$ are defined as the average number and the average sum $p_{T}$ of all the charged particles divided by $\eta-\phi$ space area $\left(2 \times \frac{2 \pi}{3}\right)$, respectively. The trigger jet $p_{T}$ is greater than 5 $\mathrm{GeV}$. The underlying events are calculated in an integrated $\eta$ range by region method. However, the $\eta$ dependence appears in the off-axis method since we define the pseudorapidity of two cones the same as the jets. From the comparison, results from both methods can roughly be consistent. So we can conclude that the effects from underlying events show small dependence on $\eta$.

We can also compare the results with those measured from STAR by using the region method. The underlying events are studied in $p+p$ collision at $\sqrt{s_{N N}}=200 \mathrm{GeV}$ from STAR [6], the c.m.s energy is comparable with our energy at EIC $\left(\sqrt{s_{N N}}=141 \mathrm{GeV}\right)$. Compared with Fig. 7 of [6], the multiplicity density of our results is shown Fig. 9(a), which is slightly lower than the STAR results. The mean $p_{T}$ of charged tracks which can be obtained from Fig. 9 (b) is close to the results from STAR. 


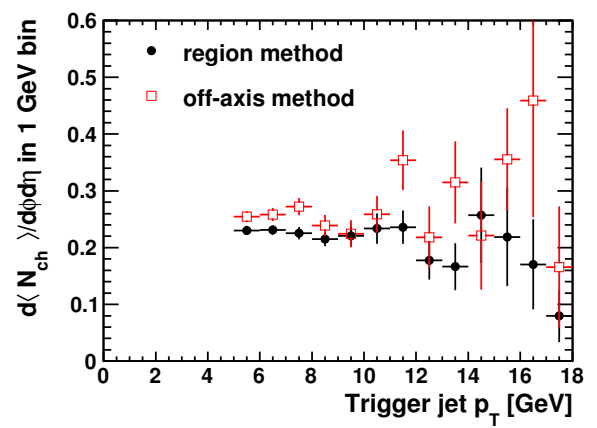

(a)

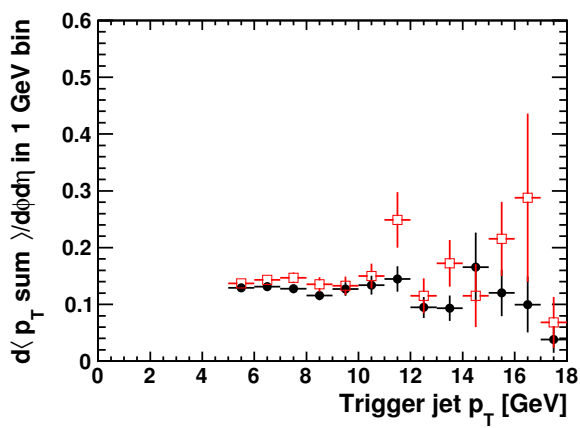

(b)

Figure 8: (a) The average number of charged particles (region method: $p_{T}>250 \mathrm{MeV},-1<\eta<1$ and $60^{\circ}<|\Delta \phi|<120^{\circ}$, the trigger jet $-1<\eta<1$; off-axis method: $p_{T}>250 \mathrm{MeV}$ and $-1<\eta<1$ ) density as a function of the transverse momentum of the trigger jet $\left(p_{T}>5 \mathrm{GeV}\right)$. (b) The average scalar $p_{T}$ sum density of charged particles (region method: $p_{T}>250 \mathrm{MeV},-1<\eta<1$ and $60^{\circ}<|\Delta \phi|<120^{\circ}$; off-axis method: $p_{T}>250 \mathrm{MeV}$ and $\left.-1<\eta<1\right)$ as a function of the transverse momentum of the trigger jet $\left(p_{T}>5\right.$ $\mathrm{GeV})$.

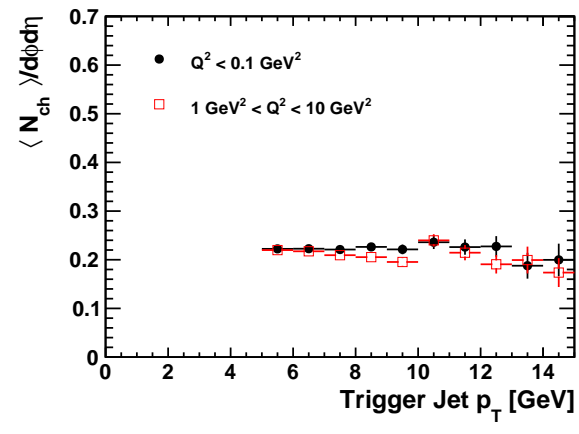

(a)

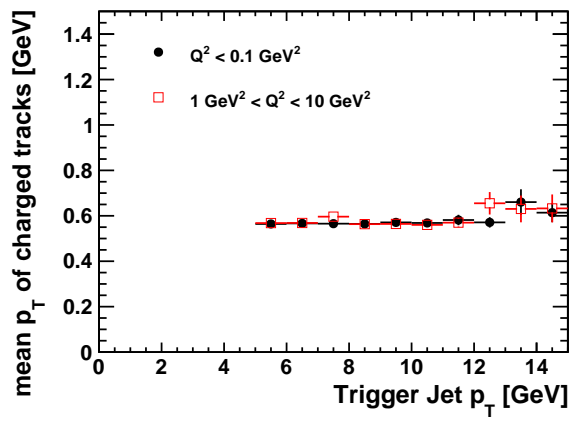

(b)

Figure 9: (a) The number density of charged particles versus the trigger jet $p_{T}$. (b) The mean $p_{T}$ of the charged particles versus the trigger jet $p_{T}$.

\section{References}

[1] A. Accardi et al., arxiv: 1212.1701; E.C. Aschenauer et al., arxiv: 1409.1633

[2] T. Sjostrand, S. Mrenna and P. Z. Skands, JHEP 0605, 026 (2006)

[3] T. Affolder, et al., (CDF Collaboration), Phys. Rev. D 65, 092002 (2002)

[4] Z. Chang, PhD thesis, https://drupal.star.bnl.gov/STAR/files/Zilong_Chang_TAMU_Thesis.pdf

[5] B. Abelev, et al. (ALICE Collaboration), Phys. Rev. D 91, 112012 (2015)

[6] J. Bielcikova (STAR Collaboration). Jet reconstruction and underlying event studies in $\mathrm{p}+\mathrm{p}$ and $\mathrm{d}+\mathrm{Au}$ collisions from STAR, arXiv:1107.4891 